\title{
OPTICAL INTERFEROMETRY IN THE MULTI-SPECKLE MODE
}

\author{
T. REINHEIMER, K.-H. HOFMANN AND G. WEIGELT \\ Max-Planck-Institut für Radioastronomie \\ Auf dem Hügel 69, 5900 Bonn 1, Germany
}

\begin{abstract}
We have studied interferometric imaging in the multi-speckle mode by computer simulations. From various simulated data sets diffraction-limited images were reconstructed by the speckle masking method and the iterative building block method. The reconstructed images show the dependence of the signal-to-noise ratio on photon noise.
\end{abstract}

\section{Experimental Results}

We have studied interferometric imaging in the multi-speckle mode by computer simulations (Reinheimer et al. 1992). In the computer experiment shown in Fig. 1 a pupil function (Fig. 1a) similar to the ESO VLT Interferometer (four 8-m telescopes), geographic latitude $-24^{\circ}$, declination $-70^{\circ}$, maximum zenith angle of $60.7^{\circ}$, and data recording at 15 different rotation angles of the earth during 9.3 hours observing time were simulated. Fig. 1b shows the uv-coverage of the experiment. Fig. 1c is the object, a close triple star (separation between the two closest stars: 0.0042 " for $\lambda=700 \mathrm{~nm}$ and $100 \mathrm{~m}$ interferometer diameter). Fig. $1 \mathrm{~d}$ shows one of the generated point source interferograms with simulated seeing corresponding to a Fried parameter $r_{0}=2 \mathrm{~m}$. The interferograms consist of about 10 speckles with interference fringes in each speckle. Speckles with fringes were obtained since many turbulence cells in front of each telescope were simulated (multi-speckle mode). Fig. 1e is one of the 48000 generated interferograms of the triple star after injection of photon noise corresponding to $\sim 300$ photoevents/frame. From the simulated VLTI interferograms the ensemble average bispectrum was derived at those positions in the 4-dimensional bispectrum space where the bispectrum transfer function $\left\langle P^{(3)}(u, v)\right\rangle$ was greater than zero. The large gaps in the uv-plane of the VLT Interferometer cause large gaps in $\left\langle P^{(3)}(u, v)\right\rangle$. From the obtained bispectrum a diffraction-limited image of the object was reconstructed by the iterative building block method. Fig. $1 \mathrm{f}$ is the diffraction-limited (resolution: $0.0018 ", \lambda=700 \mathrm{~nm}$, $100 \mathrm{~m}$ baseline) image reconstructed from the 48000 interferograms (300 photoevents/interferogram) by speckle masking (Weigelt 1977; Weigelt\&Wirnitzer 1983; Lohmann et al. 1983) and the iterative building block method (Hofmann\&Weigelt 1990 , 1992). Fig. 2b shows the diffraction-limited reconstruction of the triple star for $\sim 100$ photoevents/interferogram (same parameters as for the experiment shown in Fig. 1). 100 photoevents/interferogram correspond to magnitude 14.8 for four $8-\mathrm{m}$ telescopes, $20 \mathrm{msec}$ exposure time per interferogram, $2 \mathrm{~nm}$ filter bandwidth and $10 \%$ quantum efficiency of detector plus optics.

Fig. $3 \mathrm{a}$ is the object (galaxy; diameter $\approx 0.009$ " for $\lambda=700 \mathrm{~nm}$ and $100 \mathrm{~m}$ baseline) of a second experiment. Fig. $3 \mathrm{~b}$ shows the diffraction-limited image reconstructed from 48000 interferograms with photon noise corresponding to $\sim 5000$ photoevents/frame (same parameter as for the experiments shown in Fig.1 and 2). 


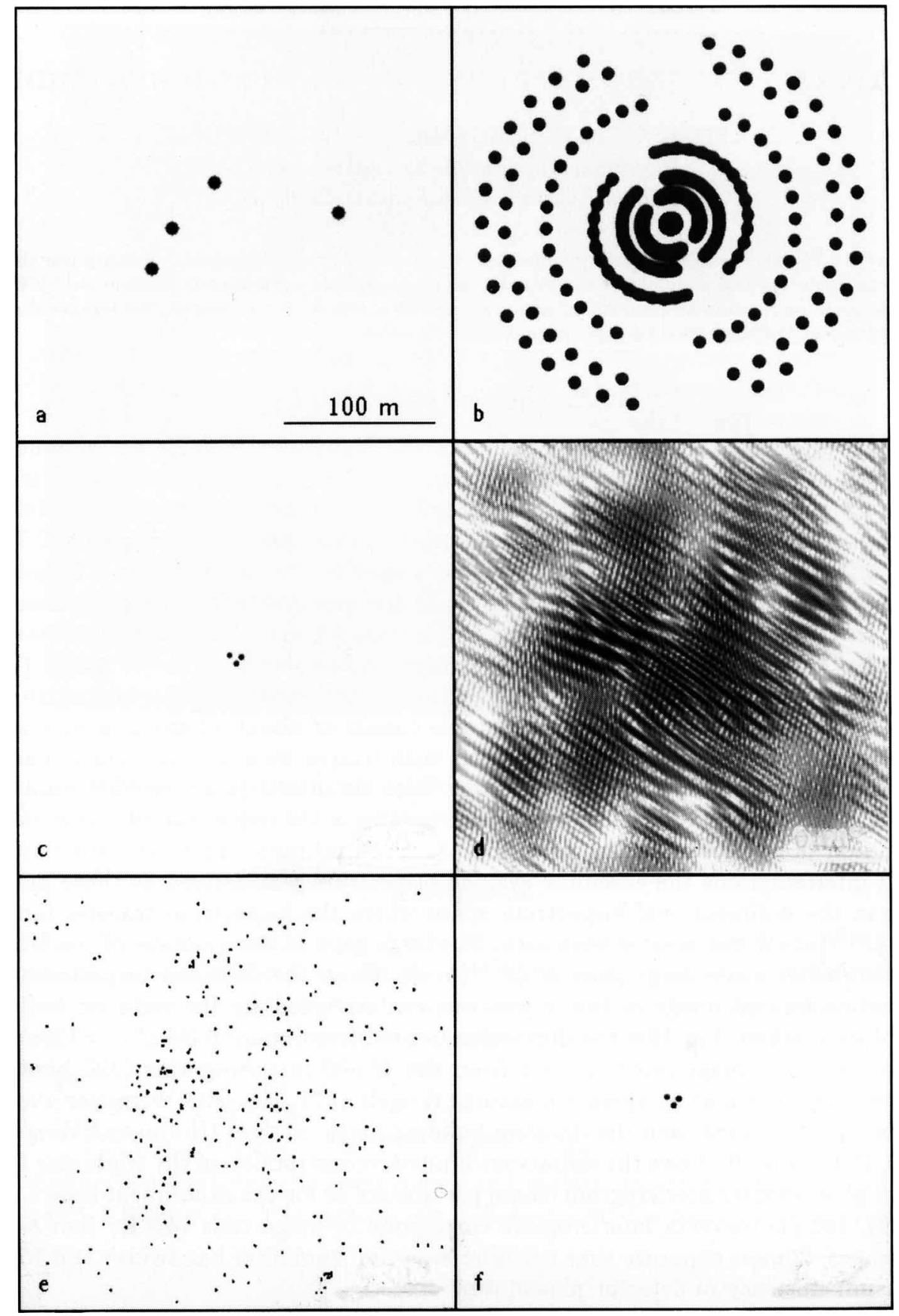

Fig. 1. Computer simulation of optical long-baseline interferometry with the ESO VLT Interferometer (four 8-m telsecopes) in the multi-speckle mode. The diffraction-limited image (f) was reconstructed from 48000 VLTI interferograms with photon noise of $\sim 300$ photoevents/interferogram (see text). 


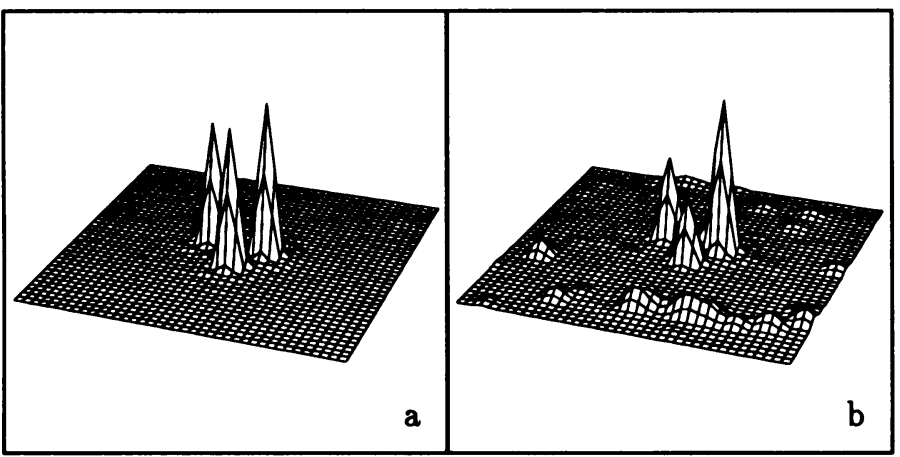

Fig. 2. Same computer simulation as described in Fig. 1, but photon noise of 100 photoevents/interferogram: (a) theoretical object, (b) diffraction-limited reconstruction derived from 48000 VLTI interferograms.

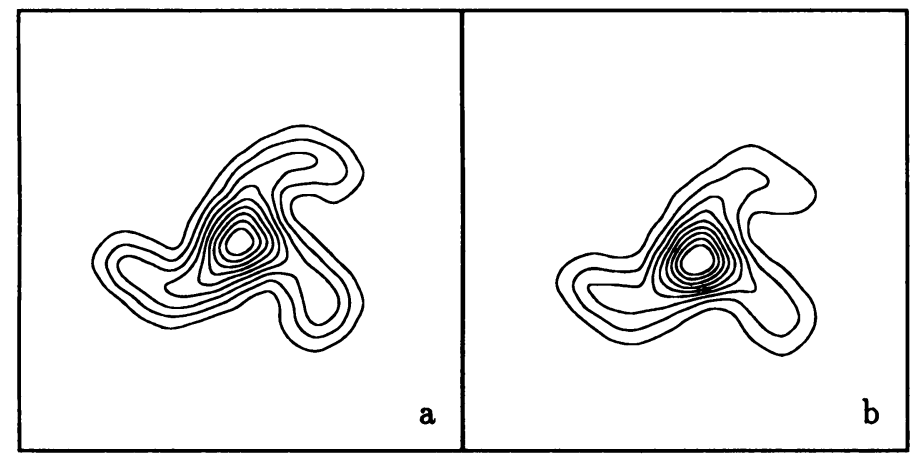

Fig. 3. Same computer simulation as described in Fig. 1, but extended object: (a) theoretical object, (b) diffraction-limited reconstruction derived from 48000 simulated VLTI interferograms with photon noise of $\sim 5000$ photoevents/interferogram.

\section{References}

Hofmann K.-H., Weigelt G.: 1990, 'Image Reconstruction from the Bispectrum Using an Iterative Algorithm, Applications of the Method to Astronomical Objects', in: Optics in Complex Systems, ICO 1990, eds. F. Lanzl, H.-J. Preuss, G. Weigelt, Soc. Photo-Opt. Instr. Eng. 1319, 444

Hofmann K.-H., Weigelt G.: 1992, 'The Building Block Method: Image Reconstruction from the Bispectrum using an Iterative Algorithm', in: High-Resolution Imaging by Interferometry II, 14-18 Oct. 1991, eds. Beckers J.M., Merkle F., European Southern Observatory, Garching, Germany, p. 193

Lohmann A.W., Weigelt G., Wirnitzer B.: 1983, Appl. Opt. 22, 4028

Reinheimer T., Weigelt G.: 1987, $A \& A$ 176, L17

Reinheimer T., Hofmann K.-H., Weigelt G.: 1992, 'Computer Simulations of Interferometric Imaging with the VLT Interferometer', in: High-Resolution Imaging by Interferometry II, 14-18 Oct. 1991, eds. Beckers J.M., Merkle F., European Southern Observatory, Garching, Germany, p.827

Weigelt G.: 1977, Optics Commun. 21, 55

Weigelt G., Wirnitzer B.: 1983, Optics Lett. 8, 389 